\title{
Kenar, Sis ve Bulut Bilişimin IoT Açısından İncelenmesi
}

\author{
Muhammet Tay ${ }^{1}$, Arafat Şentürk ${ }^{2 *}$

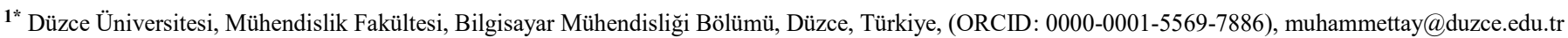 \\ 2* Düzce Üniversitesi, Mühendislik Fakültesi, Bilgisayar Mühendisliği Bölümü, Düzce, Türkiye (ORCID: 0000-0002-9005-3565), arafatsenturk@duzce.edu.tr
}

(International Conference on Design, Research and Development (RDCONF) 2021 - 15-18 December 2021)

(DOI: 10.31590/ejosat.1040133)

ATIF/REFERENCE: Muhammet, Tay. \& Arafat, Şentürk. (2021). Kenar, Sis ve Bulut Bilişimin IoT Açısından İncelenmesi. Avrupa Bilim ve Teknoloji Dergisi, (32), 68-75.

\section{$\ddot{\mathbf{O z}}$}

Nesnelerin İnterneti (IoT) alanında yaşanan büyük gelişmeler ve internete bağlı nesne/cihaz sayısında artış hızla devam etmektedir. Bu artışla birlikte verinin analiz edilmesi, depolanması ve anlamlandırılması gibi kavramların önemi gitgide artırmaktadır. Bu artış aynı zamanda çözülmesi gereken bazı problemleri de ortaya çıkarmaktadır. Bu problemlerden hız, bant genişliği ve gecikmeye çözüm olması düşünülen yeni nesil hücresel ağ teknolojisinin $(5 \mathrm{G})$ beklenen iyileştirmeyi sağlayabilmesi için yazılım ve donanım çalışmalarına ihtiyaç duyulmaktadır. Yeni nesil teknolojinin ihtiyaçlarına cevap verebilme ve artan IoT cihazları ile ortaya çıkan verinin analizi ve son kullanıcı gereksinimlerinin karşılanması için Sis, Kenar ve Bulut Bilişim (KSB) teknolojileri oldukça önemlidir. Gelişen ve büyüyen bir ağ yapısına sahip olan IoT her geçen gün mevcut ihtiyaçların yeniden ele alınmasını gerektirmektedir. Bu büyümeye paralel olarak problemlerin üstesinden gelmek için birçok araştırmacı bu alanda çalışmaktadır. Yapılan literatür çalışmasına göre, merkezileştirilmiş ağ yapısından çok, dağıtılmış ağ yapısı ve KSB teknolojisinin kullanılması gecikme, enerji tasarrufu, bant genişliği için çözüm olduğu görülmüştür. Bu çalışmada KSB kavramları ile IoT alanında sağlamış oldukları çözümlere değinilerek son yenilikler ele alındı. Ayrıca hala devam eden problemlerden bahsedilerek KSB teknolojileri IoT ile karşılaştırmalı olarak analiz edilerek elde edilen sonuçlar dile getirildi.

Anahtar Kelimeler: IoT, Kenar Bilişim, Sis Bilişim, Bulut Bilişim.

\section{Study of Edge, Fog, and Cloud Computing in Terms of IoT}

\begin{abstract}
Major developments in the field of the Internet of Things (IoT) and the increase in the number of objects/devices connected to the Internet continue rapidly. With this increase, the importance of concepts such as data analysis, storage, and interpretation is increasing. This increase also raises some problems that need to be solved. Software and hardware studies are needed for the next-generation cellular network technology $(5 \mathrm{G})$, which is thought to be a solution to speed, bandwidth, and latency, to achieve the expected improvement. Edge, Fog and Cloud Computing (EFC) technologies are very important to respond to the needs of the new generation technology and to analyze the data emerging with the increasing IoT devices and meet the end-user needs. Having a developing and growing network structure, IoT requires reconsideration of current needs day by day. In parallel with this growth, many researchers are working in this area to overcome the problems. According to the literature study, it has been seen that the use of distributed network structure and EFC technologies rather than centralized network structure is a solution for the delay, energy-saving, and bandwidth. In this study, the latest innovations were discussed by referring to the concepts of EFC and the solutions they have provided in the field of IoT. In addition, the ongoing problems were mentioned and the results.
\end{abstract}

Keywords: IoT, Edge Computing, Fog Computing, Cloud Computing.

*Sorumlu Yazar: arafatsenturk@duzce.edu.tr 


\section{Giriş}

IoT akıllı ev, havacılık sistemleri, çevre, sağlık uygulamaları, lojistik, giyilebilir teknolojiler, araç takip sistemleri ve endüstriyel uygulamalar başta olmak üzere hayatın her alanında karşımıza ç1kmaktadır (Atzori et al., 2010; Stankovic, 2014; Van Os et al., 2015; Zanella et al., 2014). Bu denli geniş bir kullanım alanı bulunan algılayıcıların veri iletimi ve alımı için karşılaşılan bazı zorluklar bulunmaktadır (Huang et al., 2014). Bu alanda yaşanan büyük gelişmeler ile internete bağlı olan cihaz sayısı yirmi milyonu geçmişken bu cihazların oluşturmuş olduğu muazzam veri ile başa çıkmak daha da zor hale gelmektedir (IoT Devices in Use Worldwide 2009-2020 | Statista, 2020).

Yeni nesil hücresel ağ $(5 \mathrm{G})$ teknolojisinin hayata geçmiş olması ve sunmuş olduğu avantajlar sayesinde internete bağlı olan cihaz sayısının artması ön görülebilir bir durumdur(Gupta \& Jha, 2015). 5G teknolojisinin en büyük avantajlarından olan bant genişliği, $1 \mathrm{~ms}$ (Milisaniye) gecikme ve 10 Gbps (Gigabayt Per Second) veri transfer hizı bulunmaktadır(Andrews et al., 2014). IoT alanında kullanılan algılayıcıların $5 \mathrm{G}$ hücresel ağ yapısına entegrasyonu ve avantajlarından yaralanabilmesi için gerekli yazılım ve donanım ihtiyacının karşılanması gerekmektedir. Özellikle gecikme duyarlı sistemlerin (sağlık alanındaki algılayıcılar, araçların interneti vs.) üretmiş oldukları verilerin analizini yapmak hayati nitelik taşımaktadır. Algılayıcı verilerinin gerçek zamanlı işlenmesi, gecikme duyarlı olması açısından KSB cihazları, çözüm olarak kullanılmaktadır (A. Botta et al., 2015; Shi et al., 2016).

$\mathrm{Bu}$ çalışmada IoT ağlarda kullanılan Kenar, Sis ve Bulut Bilişim sistemlerinin kullanım alanlarından bahsedilmiştir. Ayrıca, IoT'de kullanım alanı ve niteliğine bağlı olarak karşılaştırılmıştır. Kenar, Sis ve Bulut Bilişimin IoT ile etkileşiminden ve kullanım amacına değinilerek literatürdeki durumları analiz edilmiştir. Bu çalışma ile bahsedilen alanlardaki son yenilikler ele alınarak hala devam eden problemler ve elde edilen sonuçlar dile getirildi.

\section{IoT'de Kenar, Sis ve Bulut Bilişim Sistemleri}

IoT elektronik ve yazılım araçları kullanılarak nesnelerin veri iletimi veya alımını sağlayan ağ bağlantısıyla gömülü fiziksel cihazların, araçların, binaların ve diğer öğelerin ağı olarak ifade edilebilir(Atzori et al., 2010). IoT ağlarda internet bağlantısı için bir IP adresi içeren ve internete bağlı diğer cihaz veya sistemler arasında meydana gelen iletişimi ifade eder. Nesnelerin İnterneti, temelde fiziksel dünya ile bilgisayar sistemleri arasında mevcut ağ altyapısı üzerinden entegrasyon ve veri alışverişine izin veren mimari bir çerçevedir (Khurpade, 2018; O’Dea, 2020).

Artan IoT cihaz sayısı ve bu cihazların oluşturmuş olduğu büyük veri beraberinde bazı problemleri getirmektedir. Büyük verinin anlamlandırılması, yorumlanması veya depolanması için yazılım ve donanım alanlarında bazı iyileştirmelere ihtiyaç duyulmaktadır. Ayrıca bazı IoT cihazlarının kritik görevler üstleniyor olması veri transferinin ya da analizinin gecikme ve tıkanıklık olmaksızın yapılması gerekmektedir. Tamda bu noktada hesaplama sistemleri önemli bir rol oynayabilir. Bulut sistemlerle e-ISSN: 2148-2683 entegre olarak çalışan Kenar ve Sis Hesaplama sağlık, akıllı ulaşım gibi kritik görevdeki IoT'de etkili çözümler üretebilir (ElSayed et al., 2017; High, 2019; Shahzadi1 \& , Ambreen Niaz1, Mudassar Ali1, *, Muhammad Naeem2, Joel J.P.C. Rodrigues3, 4, 5, 6, Farhan Qamar1, 2019; Shi et al., 2016b; Yu et al., 2017).

Acil durum, sağlık ve gecikme duyarlı sistemler veri transferinde gerçek zamanlı çalışmaya yakın cevaplar dönülmesi gereken sistemlerdir. Bu noktada Nesnelerin Bulutu (Cloud of Things (CoT)) devreye girerek gerçeğe yakın cevap dönülmesi konusunda etkili çözümler sunulabilir(Aazam \& Huh, 2015). Bu etkili çözümlerin başarımı için hiyerarşik olarak Bulut Sistemler ile IoT arasında bulunan Kenar Bilişim (KB) ve Sis Bilişim (SB) önemli bir rol oynayabilir.

KSB sistemleri, büyük veri merkezi bulutu ile IoT nesneleri arasında çalışır ve amacı kaynakları yönetmek, veri filtrelemesi, ön işleme ve güvenlik olarak sıralanabilir(Banka1, 2019; Pooyan Habıbı Et Al., 2020). KSB gecikme, içerik analizi ve QoS (Quality of Service) parametrelerinin iyileştirilmesi için kullanılmaktadır. Veri analizi temelde daha hızlı karar verme, büyük veririnin işlenmesi, gerçeğe yakın zamanda cevap verme, bant genişliği, maliyet düşüklügü ve güvenlik alanlarında oldukça etkilidir. Bazı Kenar ve Sis yazılım platformları AWS Greengrass, EdgeX, Cisco FogDirector, and Cisco IOx gerçek zamanlı IoT sistemleri için Bulut Bilişim (BB) tam olarak çözüm üretememektedir. KSB IoT verilerinin buluta aktarılırken eş zamanlılık önemli bir problemdir. Tam bu noktada BB gibi çalışan KB ve SB'de çalışabilen makine öğrenmesi algoritmaları devreye girmekte ve verinin hem hızlı analizi hem de gerçeğe yakın bir zaman diliminde senkronizasyonun sağlanması gerekmektedir(Banka1, 2019). NCS (İntel Neuro Compute Stick) bu sorunlara çözüm olarak sunulmaktadır. Tam bu noktada eğitilen algoritmalar bu işlemci sayesinde daha etkili çalışacağ düşünülmektedir.

\subsection{Bulut Bilişim}

BB bilgi işlem altyapısının merkezileştirilmesini sağlamak için gerekli donanım ve yazılımların kullanıldığ 1 ve bireysel kaynak maliyetinin azaltılarak verinin merkezi bir yerde tutulmasını sağlayan sistemler bütünüdür (Hayes, 2008; Vaquero et al., 2008). BB'nin genel kabul görmüş bir açıklaması bulunmasa da dağıtık bilgisayarlar kümesi olarak nitelendirilebilir (Gong et al., 2010; Grossman, 2009), (Sultan, 2010). Bir diğer bakış açısı ile BB herhangi bir bilgisayar organizasyonunun güvenlik duvarı dışından aynı nitelikte kullanılması olarak ifade edilebilir (Knorr \& Gruman, 2011). Bir diğer ifade ile sunucu, depolama, veri tabanı, ağ, yazılım ve yapay zekanın bir arada sunulduğu merkezi bir bilgi işlem hizmetidir (Grossman, 2009; Hayes, 2008; Vaquero et al., 2008). BB kullanım alanına bağlı olarak maliyetin düşürülmesi, işlem hızı, performans, veri kaybın engellenmesi, güvenlik gibi alanlarda oldukça etkili çözümler sunmaktadır. BB'de kullanılan servisler dört kategoriye ayrılmaktadır: Infrastructure as a service (IaaS), Platform as a service (PaaS), serverless ve Software as a service (SaaS) (Chinthas et al., 2014; Mell \& Grance, 2011). Bu kategorilerin kapsamı Şekil 1'de görüldüğü gibi sıralanabilir.

IaaS: BB'nin en temel kategorisi olarak Bilgi İşlem altyapısında bulunan sunucu, sanal makine, depolama, ağ ve işletim sistemlerinin kullanılmasıdır. Bu kategorideki hizmetler 
talepte bulunanın ihtiyacı kadar hizmet sunmaktadır (Chinthas et al., 2014).

PaaS: Uygulama geliştirmek, test etmek ve yönetmek gibi hizmetlerin sunulduğu servistir. $\mathrm{Bu}$ service uygulama geliştiricileri için ihtiyaç duyulan sunucu, depolama, ağ ve veri tabanı arka planda geliştiriciden bağımsız yapmaktadır. Bu da mobil ya da web geliştiricileri için oldukça avantaj sağlamaktadır (Chinthas et al., 2014; Vaquero et al., 2008).

Serverless: Sunucusuz servis olarak adlandırılan bu servis ile uygulama geliştiricileri için gerekli olan altyapı çalışmalarını otomatik olarak oluşturarak uygulamaların daha hızlı kullanıma sunulmasını sağlar. Bir diğer avantajı ise uygulama yönetiminin daha etkili kullanılmasına olanak tanımasıdır (W. Wang, 2016).

SaaS: Geliştirilen uygulamalara tarayıcıdan erişimini sağlayan ve güncelleme ve güvenlik gibi yenilemelerin hızlıca yapılabildiği bir servistir (W. Wang, 2016).

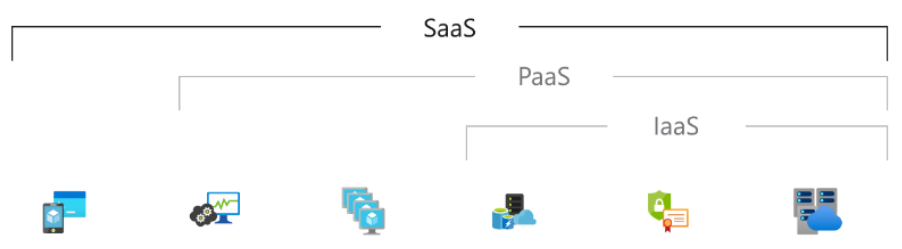

Şekil 1 Bulut servislerin kapslamı (Mell \& Grance, 2011; Microsoft, 2019)

BB'nin mimari yapısı 5 temel faktör olan geliştirici, denetleyici, taşıyıcı, sağlayıcı ve tüketiciden oluşmaktadır (Grossman, 2009; Hayes, 2008; Knorr \& Gruman, 2011).

- Servis sağlayıcı: ilgili taraflara hizmet sağlayan bir kuruluştur.

- Tüketici: Bulut Sağlayıcısının hizmetlerden yaralanan kişi ya da kurumdur.

- Servis geliştirici: Servis sağlayıcı ile tüketici arasındaki düzenlemeleri yapar ve hizmetlerin kullanımını, performansını ve dağıtımını yönetir.

- Servis taşıyıcı: Sağlayıcı ile tüketici arasındaki hizmet bağlantısını sağlamaktadır.

- Denetleyici: Sitem, güvenlik ve alt yapının sorunsuz çalışmasını sağlar.

\subsection{Sis Bilișim}

CISCO'ya göre SB son kullanıcı ile BB arasındaki hesaplama, depolama ăg servisleri gibi hizmetlerin sağlayan ancak ağın kenarına yakın olmayan yüksek seviyeli sanallaştırılmış bir platformdur. SB sadece nesneler için kullanılmaz heterojen yapıdaki son kullanıcıları da kapsar. Bir diğer değişle SB merkezi bir sistem olan BB'nin dağıtılmış ve sınırlandırılmış halidir. Buna göre Sis cihazları Bulut ile düğümler arasında herhangi bir yere konumlandırılabilir. SB BB yerine geçen bir sistem değil BB ile Kenar Bilişimi destekler ve geliştirir (Gazori et al., 2020; Hamid Reza Arkian, Abolfazl Diyanat, 2017).

SB alanında literatürde dile getirilen mimarilerden ikisi incelenmiş. Her iki mimaride hiyerarşik bir yapıya sahip olduğu görünmüştür. $\mathrm{Bu}$ mimarilerin hiyerarşik bir yapıya sahip olduğu görülmüştür. Bu mimarilerden ikisi olan Üç katmanlı mimari ve $\mathrm{N}$ katmanlı mimari incelenmiştir.

Üç Katmanlı Mimari: Bu mimariye göre en alt katmanda IoT yer alır. Bu katmada IoT cihazları, algılayıcılar, araçlar, akıllı telefonlar ve tabletler bulunur. Bu katmandaki cihazlar genel anlamda bir üst katman olan Sis ve Bulut katmanlarına Şekil 2'de görüldüğü gibi veri gönderir. Bu veriler depolama ya da işlenme niteliği taşıyan verilerden oluşur. Orta katmanda ise SB bulur ve bu katmanda alt katmandan gelen verilerin işlenmesi, iletilmesi ve geçici depolanması gibi hizmetler sunar ve son katmanda ise BB bulunur. BB teknolojileri de SB gibi depolama, analiz, hesaplama gibi hizmetleri SB'ye göre daha kapsamlı bir şekilde sunar (Aazam et al., 2016; Shah-Mansouri \& Wong, 2018).

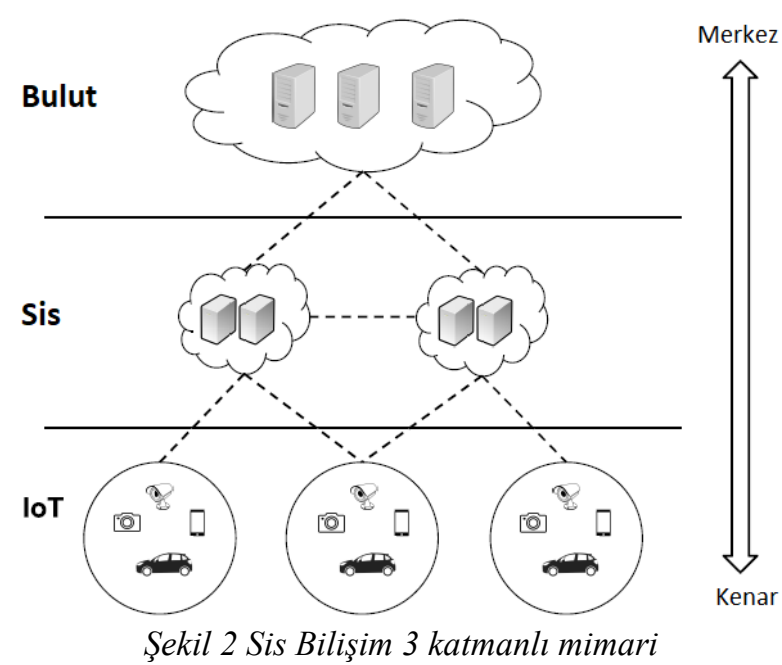

OpenFog N-Aşamalı Mimari: Bu mimaride üç katmandan oluşmaktadır. Ancak bir önceki başlıkta belirtilen Üç Katmanlı Mimariden farklı olarak SB üç farklı rol ve nitelikte çalışmaktadır. Buna göre orta katmanda bulunan SB üç aşamalı olarak IoT ve BB'nin iletişiminini düzenler. Bu aşamalar alt aşama, orta aşama ve üst aşama olarak ifade edilebilir. Alt aşamada bulunan SB'ler IoT ile veri iletimi yaparken orta aşamadaki SB'ler ise kenara yakın olan SB ile buluta yakın SB arasında köprü görevi görür. Son aşamadaki SB'ler ise doğrudan BB ile etkileşim halindedir. Bu yaklaşıma göre orta katmanda çoklu SB sistemleri kullanılması ön görülmektedir. Çoklu SB kullanımı SB'ler arasında etkili bir iş birliği ile yoğun IoT'lerde daha etkili sonuçlar alınması sağlanmıştır (Shahzadi1 \& , Ambreen Niaz1, Mudassar Ali1, *, Muhammad Naeem2 , Joel J.P.C. Rodrigues3, 4, 5, 6 , Farhan Qamar1, 2019).

\subsection{Kenar Bilişim}

Kenar Bilişim (KB) hesaplama ihtiyaçlarının ağın kenarında yapılmasını sağlayan teknolojidir (El-Sayed et al., 2017). KB'nin amacı BB hizmetlerinin ağın kenarına konumlandırılması olarak ifade edilebilir. İstemci verilerinin mümkün olduğunca kaynağa yakın olarak işlenmesi yöntemine dayanmaktadır. Bir diğer ifade ile BB'nin optimize edilmiş hali olarak düşünülebilir. KB zaman ve kaynaktan tasarruf etmemize olanak tanımaktadır (El-Sayed et al., 2017; Y. Liu, M. Peng, G. Shou, 2020).

KB'nin uygulanması bağlı olan cihaz tiplerine, iletişim protokollerine ve kullanılacak servislere göre değişmektedir. Bu uygulamalardan en çok kullanılan Cloudlet Computing (CC) ve Mobil Kenar Hesaplama (MKH)'dır (Qi \& Tao, 2019; Sahni et al., 2017; Tuysuz, 2019; Wang et al., 2019). 
CC: Uygulama alanı mobil yük dengelemeye dayanmaktadır. Buna göre mobil cihazlar depolama ve hesaplama ihtiyaçları için en etkili yük dengelemeyi kullanarak enerji tasarrufu, yaşam döngüsü ve maliyetin düşürülmesi hedeflenmektedir (Yu et al., 2017).

MKH: Uygulamaya göre Kenar Cihaz (KC) BB'nin sağlamış olduğu hizmetlerin optimize edilmiş haliyle bağlı olan cihazlara hizmet vermektedir. MKH ile son kullanıcıya lokasyon veya içeriğe göre hizmet vermekte ve bu da düşük gecikme ve yüksek bant genişliği gibi avantajlar sunmaktadır (Yu et al., 2017). Ancak MKH'nin bazı uygulama alanlarında hala etkili çözümler olmadığı görülmektedir. Buna göre heterojen bir ağ yapısında MHK'nin uygulanabilmesi için framework ihtiyacı doğmaktadır (Shi et al., 2016b).

\subsection{KSB alanında yapılan çalışmalar}

KSB'nin IoT alanında daha etkili kullanılması için bazı zorluklar bulunmaktadır. Çalışmanın bu bölümünde bahsedilen zorluklar sınıflandırılarak beş başlık altında sıralandı ve yapılan çalışmalarda bu zorluklara getirilen çözümler incelendi. Bunlar; ölçeklenebilirlik ve hareketlilik, yük boşaltma (offloading), yorumlanabilirlik, enerji tüketimi ve planlama sinıflarından oluşmaktadır (Mostafa Ghobaei-Arani, Alireza Souri, 2019). Bölümün devamında iler ki çalışmalara ışık tutması açısından bu zorluklar hakkında daha önce yapılmış çalışmalardan bahsedilecektir.

Ölçeklenebilirlik ve hareketlilik: SB'de kullanılan cihazların oyun teorisine göre kullanımının belirlenmesi ve gereksiz verilerin çalışma alanında çıkarılması Shah-Monsouri ve arkadaşları (Shah-Mansouri \& Wong, 2018) tarafindan hedeflenmiştir. Bu hedef doğrultusunda hem hız hem de enerji konusunda iyileştirme yapıldığı ifade edilmektedir. Ayrıca hesaplama kaynaklarının çevrimiçi kullanılması hedeflenmiştir (Shah-Mansouri \& Wong, 2018).

S.P. Abedin ve arkadaşları (S. F. Abedin, M. G. R. Alam, S. M. A. Kazmi, N. H. Tran, 2019) mobile Kenar hesaplama alanında önemli eksikler bulunduğu ifade ettikleri çalışmada kablosuz spektrumu mobil cihazlar arasında hesaplama için dağıtılmasını öngörülmüş̧ür. Bu sayede $5 \mathrm{~g}$ bağlamında iyileştirme sağlandığı görülmektedir.

Bir diğer çalışma ise Multimedia sensing as servise (MSaaS) INN IoT alanında kullanılan video streaming işleminin büyük veri kategorisinde incelenerek kaynakların doğru tahsis edilmesi için gerekli optimizasyonu sağlamaktadır (W. Wang, 2016). Buna göre Qoalitiy of Experience (QoE) konusunda iyileştirme yapıldığ1 görülmektedir. Ayrıca uygulama katmanında veri çeşitliliği esneklik sağlamaktadır (W. Wang, 2016).

Yük Dengeleme (Offloading): Bu alanında yapılan çalışmalar ise genel anlamda cevap verme sürelerinin düşürülmesi hedeflenmiştir.

Kaynakların tahsisi ve cevap süresinin düşürülmesine yönelik H. R. Arkian (Hamid Reza Arkian,Abolfazl Diyanat, 2017) ve arkadaşları tarafından MIST algoritması önerilmiştir. $\mathrm{Bu}$ algoritma ile sis hesaplaması tabanı bir planlama sistemidir.
Simülatör olarak CloudSim kullanılmış (Hamid Reza Arkian, Abolfazl Diyanat, 2017).

Yine bir diğer çalışmada verinin işlenmesi ve anlamlı hale getirilmesi için merkezi bir sunucuya göndermek yerine (bulut) kenar ya da sis hesaplama cihazlarına gönderen Kenar yoğun adında yeni bir paradigma önerilmiştir (Sahni et al., 2017). Sağlık, akıllı ulaşım ve akılı ev sistemleri için senaryolar geliştirilerek etkinliği üzerinde çalışmalar yapılmıştır. Cihazlara yönelik görev tahsisi konusunda genetik algoritma kullanılmıştır. Gelecek çalışmalara yönelik olarak farklı hesaplama paradigmalarının entegrasyonunun sağlanması, heterojen cihazların dahil edilmesi hedeflenmiştir (Sahni et al., 2017).

Başka bir çalışmada ise DRAM algoritması ile statik kaynak tahsisi ve dinamik servis geçişi ile yük dengeleme işlemi yapılmaktadır. Geleceğe yönelik olarak dinamik servis geçişlerinin ağa olan etkisinin ve servis geçişlerinin etkisi üzerine çalışma yapılabilir önerisinde bulunulmuş (Xu et al., 2018). Bir başka çalışmada ile Sis ve Bulut arasındaki iletişim için bir mimari önerisinde bulunulmuştur.

Alsarraf ve arkadaşları (Alsaffar et al., 2016) quality of service (QoS) ve service level agreement (SLA) açısından büyük verinin dağıtılması optimizasyonu ve kaynakların tahsisi konusunda daha etkili çözümler üretilmiştir (Alsaffar et al., 2016).

S. S. Gill ve arkadaşları (S. S. Gill, P. Garraghan, 2019) kritik uygulamalarda gerçek zamanlı için kaynak tahsisi için parçacık sürü optimizasyonu algoritması kullanılmışıtır. İFogSim simülasyonu kullanılmış ve bant genişliği, enerji, gecikme ve cevap sürelerinde daha etkili olduğu görülmüştür. Geleceğe yönelik olarak ise gecikme ve enerji tüketimi çalışılabilir önerisinde bulunulmuştur (S. S. Gill, P. Garraghan, 2019).

F. Chiti (F. Chiti, 2018) IoT ağların alanında yoğun hesaplama gerektiren son kullanıcı ile sis hesaplama arasındaki görevleri yerine getiren bir algoritma önermiştir. Buna göre iletişim ve hesaplama maliyetinin en iyi şekilde kullanılması için potansiyel oyun teorisi dişsallığına bağlı bir algoritma geliştirilmiştir (F. Chiti, 2018). Bu algoritmaya göre en kötü toplam bekleme süresi, ortalama bekleme süresi ve görev başına bekleme süresi dikkate alınarak kaynak tahsisine karar verilir. Buna göre çalışma ile IoT tabanlı sis hesaplamada daha az gecikme ile veri gönderimi sağlanmıştır.

Yorumlanabilirlik: $\mathrm{Bu}$ kapsamındaki çalışmalar ele alındığında veri alışverişi ve bilgi paylamışı konuları dikkat çekmektedir. Aazam ve ardakaşları (Aazam \& Huh, 2015) Agecikme duyarlı sistemlerin ihtiyaç duyduğu hızlı cevap vermeyi çözmeye çalıştı. Bu çalışma ile kaynak tahmini, kaynak tahsisi ve maliyet konusunda iyileştirme yapılmış ve simülasyon olarak CloudSim toolkit kullanılmış. Bu çalışma ile Sis katmanında micro veri merkezleri ile iyileştirme sağlanmış (Aazam \& Huh, 2015). Daha çok heterojen cihazlar ve hareketlilik faktörünün eklenmesi alanında çalışmaların geliştirilmesi önerilmiş.

Bir başka çalışma ile sis hesaplama ile yapılması gereken işlemlerin (kaynak tahsis, veri filtreleme vs) verimli bir şeklide kullanılmasını amaçlamaktadır (Aazam et al., 2016). Bu çalışma ile birkaç seçenek üzerinden kaynakların tahsis edilmesi işlemi 
yapılmaktadır. Bu seçenekler müşterinin vazgeçme olasılı̆̆ 1 , hizmet türü ve hizmet fiyatı olarak sıralanabilir. Önerilen yöntem Crawdad real trace and Amazon EC2 de uygulanmış ve amazonun önceki verileri kullanılarak belli bir karar alınması sağlanmış ve daha iyi sonuç aldığı görülmüştür. Bu çalışma ile elde edilen sonuçlara bakıldığında ayrı servis türleri için optimal kaynakların ne olması gerektiği analizi ile kaynakların daha verimli kullanılması sağlanmış bulunmaktadır. Amazon bulut verileri dikkate alındığında en çok mobil kullanıcılara hitap eden uygulamalar (Netflix, ICloud vs) dikkat çekmektedir (Aazam et al., 2016).

Y. Liu ve arkadaşları (Y. Liu, M. Peng, G. Shou, 2020) KB' de işlenecek verilerin dağıtılmasını sağlayan bir mimari önermiş. İşlenecek verinin zamansal olarak buluta ya da kullanıcıya yakın bir sunucuda çalıștırılmasına olanak sağlamaktadır. Verinin tamamının transferi yerine ilenerek gerekli olanının iletilmesi oldukça önemli bir faktörüdür (Y. Liu, M. Peng, G. Shou, 2020).

Multi-access edge computing (MEC) son kullanıcılar için gecikmeyi azaltmayı ve MEC'in $5 \mathrm{~g}$ ve IoT bağlamında değerlendirilmesini kapsamaktadır. Derin öğrenme algoritması kullanılarak MKH' da kullanıcı deneyimlerinden yola çıkarak kaynak tahsis, hareketlilik, güvenlik ve enerji konularında ilerleme sağlanmıştır (McClellan et al., 2020). Yapay zekâ ile trafik (ağ) akış kontörlü yapılmış (M. Chen, Y. Miao, H. Gharavi, 2020). Bunun için long short-term memory (LSTM) ile eğitim yapılarak iyileştirmeye gidilmiş.

Enerji Tüketimi: Bir diğer önemli çalışma alanı olan enerji tüketimi konusunda da etkili çalışmalar yapılmış ancak hala tam anlamıyla enerji probleminin çözüme kavuşturulduğu söylenemez. KH alanında çalışan sistemler buluttan daha iyi sonuç verdiği görülmüş ve gelecek çalışma konusu olarak gerçek zamanlı optimizasyon teknikleri, kenar ve bulut arasındaki kaynak tahsisinin düzenlenmesi ve son olarak etkili planlama algoritmaları enerji konusunda fark yaratabilir (Hesham ElSayed, Sharmi Sankar, Mukesh Prasad, Deepak Puthal, Akshansh Gupta, 2018).

Heterojen sis ağlar için MEETS (maximal energy efficient task sheduling) algoritmasını öneren Yang ve arkadaşları(Yang et al., 2018) komşu spektrum ve enerji parametrelerini değerlendirilerek en uygun parametre seçimi yapılması hedeflenmiştir. $\mathrm{Bu}$ çalışma ayrıca heterojen ağ yapısına uygulanarak gelecek nesil (5g) teknolojisine de uygunluğu artırılmış ve çeşitli senaryolarda görev zamanlaması alanında etkili sonuçlar üretmiştir (Yang et al., 2018).

Planlama (Scheduling): Kenar ve sis hesaplamada en çok çalışma yapılan konuların başında gelen planlama (scheduling) alanı oldukça önemli ve gecikme başta olmak üzere birçok alanda iyileştirme sağlanabilecek bir alan olarak karşımıza çıkmaktadır.

Student Project allocation (SPA) game optimizasyonu kullanılarak kaynak tahsisinde ortak radio ve hesaplamalı kaynak tahsisine çözüm aranmıştır (Y. Gu, Z. Chang, M. Pan, 2018). Ayrica sistem performansı user-oriented cooperation (UOC) dikkate alınarak daha fazla geliştirilmiştir.

Tiscornia ve arkadaşları (Tiscornia et al., 1999) QoS ve Service Level Agreement (SLA) bağlamında iyileştirme yapılmış ve çalışma CloudSim simülatörü ile amazonun EC2 kaynak tahsisinde kullanılarak analiz edilmiş ve daha iyi sonuç ürettiği görülmüştür (Tiscornia et al., 1999). MeFoRE (Media fog kaynak tahmini) algoritmasına dayanmaktadır. Kaynaklardan vazgeçme hesaplamasına dayanmaktadır. Bu çalışma ile mikro düzeyde bedel tabanlı maliyet modeli önerilmektedir. Bu maliyet hesabı yapıldıktan sonra kaynakların tahsisi yapılmakta ve çalışma ile hem kullanıcı bazında hem de sağlayıcı bazında iyileştirme yapılmıştır Metin girmek için buraya tıklayın veya dokunun. Gelecek çalışma alanı ise önerilen yöntemin blockchain ile yapılması olarak planlanmıştır.

Bir başka çalışma ile Blockchain tabanlı networklerde derin öğrenme ile kaynak tahsis önerilmiştir. Çalışma 4 aşamadan meydana gelmektedir. Birinci aşamada öncelikle kaynak tahsisindeki sonlar tespit edilmiş, ikinci aşamada ise ekonomik ve maliyet hesabı yapılmış, üçüncü aşamada ise Sis ve Blockchain tanımlanmış ve son olarak ta derin öğrenme uygulanarak kaynak tahsisine karar verilmiştir (Nguyen Cong Luong, Yutao Jiao, Ping Wang, Dusit Niyato, Dong In Kim \& To, 2020). Ayrıca dinamik çalışan bir sisteme uygulanması hedeflenmiştir.

Önerilen bir başka çalışma ile otomatik kaynak tahsisi ve seçim işlemini yapay sinir ağı modeline uygulanarak gerçekleştirilmiştir. Bu çalışma Fog Resource Selection Services (FReSS) algoritması olarak adlandırılmıştır (Mostafa, 2020). Simülasyon CloudSim ile gerçekleştirilmiştir. Double Deep Reinforcement Learning algoritması kullanarak Sis hesaplama için daha az karmaşık ve gecikmeye dayalı bir iyileştirme yapılmıştır (Gazori et al., 2020). Karşılaştırma olarak algoritma karmaşıklığı, enerji maliyeti, gecikme, hata töleransı ve yük dengeleme parametrelerince karşılaştırılmıştır.

\section{IoT'de Kenar, Sis ve Bulut Bilişim Sistemleri}

IoT'nin yaygnlaşmasıyla birlikte Kenar, Sis ve Bulut teknolojilerinin bu alana entegrasyonu hızlanmitır. Kenar ve Sis teknolojileri genel anlamda, büyük ölçekli IoT ağlarında bilgi edinme, bilgi analizi, karar verme ve eylem uygulamasını içeren görevlerin yerine getirilmesi için etkin rol alabilirler. Bulut bilişim ise bir nevi veri depolama ve analiz etme alanı olarak düşünülebilir.

KSB temelde nesneler ile bulut arasında bulunan yazılım ve donanım araçlarıdır. Bulut sistemler ile aynı görevi üstlenmektedir. Ancak bazı uygulama alanlarında veri analizinin veya veri aktarımın gecikme duyarlı olmasından dolayı ve bulut servislerinin bu hizmetleri tam olarak yerine getirememesinden dolayı ortaya çıkmışlardır (Banka1, 2019). Bir başka değişle Sis hesaplama bulut işlemlerinin ağın kenarına (kullanıcıya en yakın olan fiziksel konum) yakınlaştırılması ve işlemlerin dağıtılması olarak ifade edilebilir. Sağlık alanında çalışan nesnelerin verilerinin gecikmeli gitmesi hayati risk taşımaktadır ve bunun gibi sistemlerde etkili çözümler sunabilir (Aazam et al., 2016; Aazam \& Huh, 2015).

Kullanım kriterine göre sınıflandırıldığında KB cihazlarının kullanılması düşük gecikme ile veri iletimi yapmaktadır. Düşük gecikme gerektiren sistemlerde kullanılması oldukça avantajlı bir durum oluşturmaktadır. Öte yandan kaynak başına işlem süresi ele alındığında BB'nin daha avantajlı olduğu görülmektedir. 
Tablo 1. IoT vs Kenar, Sis ve Bulut Bilişim (Gong et al., 2010; Hayes, 2008; Knorr \& Gruman, 2011; Shi et al., 2016b; Sultan, 2010; Vaquero et al., 2008; Yu et al., 2017)

\begin{tabular}{l|c|c|c|c}
\hline Öz Nitelik & IoT & Kenar Bilişim & Sis Bilişim & Bulut Bilişim \\
\hline Yayılım & Dağıtık & Dağıtık & Dağıtık & Merkezi \\
\hline Bileşenler & $\begin{array}{c}\text { Fiziksel } \\
\text { Cihaz }\end{array}$ & Kenar Düğümleri & Sis Düğümleri & Sanal Kaynaklar \\
\hline Depolama & Sınırlı & Sınırlı & Sınırlı & Sınırsız \\
\hline Cevap Süresi & - & Hızlı & Orta & Yavaş \\
\hline Konumlandırma & - & Kullanıcıya Yakın & Kullanıcıya Yakın & Kullanıcıya Uzak \\
\hline Gecikme & - & Düşük & Orta & Yüksek \\
\hline Kaynak Başııa işlem süresi & - & Düşük & Düşük & Yüksek \\
\hline
\end{tabular}

IoT’ nin kullanım alanına bağlı olarak elde edilen sonuçlar Tablo 1'de dile getirilmiş ve belirtilen öz nitelikler için hangi sistem daha etkili bir çözüm olabileceği belirtilmiştir.

Literatür çalışmalarından elde edilen sonuçlara göre devam eden problemler şöyle sıralanabilir:

- Mobil SB'de etkili bir cevap süresi (Okay, 2019).

- SB'de offloading için daha etkili bir makine öğrenmesi algoritmasına ihtiyaç duyulması (Okay, 2019).

- BB'de güvenlik ve hesaplama için optimize edilmiş bir enerji tasarruf politikası (De Donno et al., 2019).

- MCS (Mobile crowd sensing) cihazların birbirleri ile iletişiminin sağlanması ve paylaşım yapıla bilinmesi problemi. Bu problem temelde heterojen ağ yapısı ile ilişkilendirilmektedir (Marjanovic et al., 2018).

- Hetorjen IoT ağlarında daha etkili çalışabilecek bir KB mimarisi (El-Sayed et al., 2017).

- Hareketli bir ağ yapısına sahip olan VANET (Vechile area networks) ve MANET (Mobile ad hoc neworks) için etkili bir mobil KH önerilmesi (El-Sayed et al., 2017).

- DoS saldırılarında yaşanacak kaynak kaybından dolayı yeni ve etkin kaynak paylaşım mimarisi (El-Sayed et al., 2017).

\section{Sonuçlar}

Bu çalışmada, KSB'nın yapısı ve IoT alanında kullanımı incelenmiş̧tir. Buna bağlı olarak KSB'nin hangi alanlarda daha etkili çözümler sağlayabileceği ifade edilerek bir karşılaştırma yapılmış. Aynı zamanda, KSB alanında devam eden problemler ve bu problemlere getirilen çözümler ifade edilmiştir. Yine çalışmada SBK'nın genel kullanım alanları işlenerek bu alanda devam eden problemlere değinilmiştir. Bazı IoT ağları için kritik nitelik taşıyan düşük gecikme, depolama, cevap süresi ve işlem süresi gibi nitelikler dikkate alındığında KSB'nın oldukça avantajlı olduğu görülebilir. $\mathrm{Bu}$ da $5 \mathrm{G}$ teknolojisinin gerekliliklerini karşılaması açısından önemli bir etken olarak karşımıza çıkmaktadır. Ancak Kenar ve Sis Bilişimin yaygın bir kullanımının bulunmaması bir dezavantaj olarak görülebilir.

Bundan sonraki çalışmada MKH'in heterojen ağlarda çalışılması hedeflenmektedir. Heterojen bir IoT ağında MKH'nin daha etkili bir sonuç üreterek cevap süresinin düşürülmesi hedeflenmektedir.

\section{Kaynakça}

IoT devices in use worldwide 2009-2020 | Statista. (2020). Retrieved September 30, 2020, from https://www.statista.com/statistics/764026/number-of-iotdevices-in-use-worldwide/

Aazam, M., \& Huh, E. N. (2015). Fog computing micro datacenter based dynamic resource estimation and pricing model for IoT. In Proceedings - International Conference on Advanced Information Networking and Applications, AINA (Vols. 2015-April, pp. 687-694). https://doi.org/10.1109/AINA.2015.254

Aazam, M., St-Hilaire, M., Lung, C. H., \& Lambadaris, I. (2016). PRE-Fog: IoT trace based probabilistic resource estimation at Fog. In 2016 13th IEEE Annual Consumer Communications and Networking Conference, CCNC 2016 (pp. 12-17). https://doi.org/10.1109/CCNC.2016.7444724

Alessio Botta, Walter de Donato, Valerio Persico, A. P. (2015). Integration of Cloud Computing and Internet of Things: a Survey Alessio. Future Generation Computer Systems.

Alsaffar, A. A., Pham, H. P., Hong, C. S., Huh, E. N., \& Aazam, M. (2016). An Architecture of IoT Service Delegation and Resource Allocation Based on Collaboration between Fog and Cloud Computing. In Mobile Information Systems (Vol. 2016). https://doi.org/10.1155/2016/6123234

Andrews, J. G., Buzzi, S., Choi, W., Hanly, S. v., Lozano, A., Soong, A. C. K., \& Zhang, J. C. (2014). What will 5G be? In IEEE Journal on Selected Areas in Communications (Vol. 32, Issue 6, pp. 1065-1082). https://doi.org/10.1109/JSAC.2014.2328098

Atzori, L., Iera, A., \& Morabito, G. (2010). The Internet of Things: A survey. In Computer Networks (Vol. 54, Issue 15, pp.

2787-2805). https://doi.org/10.1016/j.comnet.2010.05.010

Banka1, G. M. | K. M. | H. (2019). Internet of Things and data analytics: A current review. Wiley.

Battula, S. K., Garg, S., Naha, R. K., Thulasiraman, P., \& Thulasiram, R. (2019). A micro-level compensation-based cost model for resource allocation in a fog environment. In Sensors (Switzerland) (Vol. 19, Issue 13). https://doi.org/10.3390/s19132954 
Chinthas, A., Rani, D., \& Ranjan, R. K. (2014). A Comparative Study of SaaS, PaaS and IaaS in Cloud Computing. In International Journal of Advanced Research in Computer Science and Software Engineering (Vol. 4, Issue 6). www.ijarcsse.com

De Donno, M., Tange, K., \& Dragoni, N. (2019). Foundations and Evolution of Modern Computing Paradigms: Cloud, IoT, Edge, and Fog. IEEE Access, 7, 150936-150948. https://doi.org/10.1109/ACCESS.2019.2947652

El-Sayed, H., Sankar, S., Prasad, M., Puthal, D., Gupta, A., Mohanty, M., \& Lin, C. T. (2017). Edge of Things: The Big Picture on the Integration of Edge, IoT and the Cloud in a Distributed Computing Environment. IEEE Access, 6, 1706-1717.

https://doi.org/10.1109/ACCESS.2017.2780087

F. Chiti, R. F. and B. P. (2018). A Matching Theory Framework for Tasks Offloading in Fog Computing for IoT Systems. Internet of Things Journal, 5(6), 5089-5096.

Gazori, P., Rahbari, D., \& Nickray, M. (2020). Saving time and cost on the scheduling of fog-based IoT applications using deep reinforcement learning approach. In Future Generation Computer Systems (Vol. 110, pp. 1098-1115). https://doi.org/10.1016/j.future.2019.09.060

Gong, C., Liu, J., Zhang, Q., Chen, H., \& Gong, Z. (2010). The characteristics of cloud computing. Proceedings of the International Conference on Parallel Processing Workshops, 275-279. https://doi.org/10.1109/ICPPW.2010.45

Grossman, R. L. (2009). Cloud Computing. http://hadoop.apache.org/core

Gupta, A., \& Jha, R. K. (2015). A Survey of 5G Network: Architecture and Emerging Technologies. In IEEE Access (Vol. 3, pp. 1206-1232). https://doi.org/10.1109/ACCESS.2015.2461602

Hamid Reza Arkian,Abolfazl Diyanat, A. P. (2017). MIST: Fogbased Data Analytics Scheme with Cost-Efficient Resource Provisioning for IoT Crowdsensing Applications. Journal of Network and Computer Applications, 82, 152-165.

Hayes, B. (2008). Cloud Computing. Communications of the ACM, 51(7), 9-11. https://doi.org/10.1145/1364782.1364786

Hesham El-Sayed, Sharmi Sankar, Mukesh Prasad, Deepak Puthal, Akshansh Gupta, M. M. (2018). Edge of Things: The Big Picture on the Integration of Edge, IoT and the Cloud in a Distributed Computing Environment. IEEE Access, 6, 1706-1717.

High, R. (2019). IBM Edge Computing. In Ibm.

Huang, H., Zhu, J., \& Zhang, L. (2014). Internet of Things (IoT): A vision, architectural elements, and future directions. In IET Conference Publications (Vol. 2014, Issue CP639, pp. 175-179). https://doi.org/10.1049/cp.2014.0680

Khurpade, J. M. (2018). A SURVEY ON IOT AND 5G NETWORK. 2018 International Conference on Smart City and Emerging Technology (ICSCET), 1-3.

Knorr, E., \& Gruman, G. (2011). What Cloud Computing Really Means. www.infoworld.com

M. Chen, Y. Miao, H. Gharavi, L. H. and I. H. (2020). Intelligent Traffic Adaptive Resource Allocation for Edge Computingbased 5G Networks. Transactions on Cognitive Communications and Networking, 6(2), 499-508.

Marjanovic, M., Antonic, A., \& Zarko, I. P. (2018). Edge computing architecture for mobile crowdsensing. IEEE
Access,

6 ,

https://doi.org/10.1109/ACCESS.2018.2799707

McClellan, M., Cervelló-Pastor, C., \& Sallent, S. (2020). Deep learning at the mobile edge: Opportunities for $5 \mathrm{G}$ networks. In Applied Sciences (Switzerland) (Vol. 10, Issue 14). https://doi.org/10.3390/app10144735

Mell, P., \& Grance, T. (2011). The NIST Definition of Cloud Computing Recommendations of the National Institute of Standards and Technology.

Microsoft. (2021). Microsoft Azure. Retrieved September 16, 2021, from https://azure.microsoft.com/enus/overview/what-is-saas/

Mostafa Ghobaei-Arani, Alireza Souri, A. A. R. (2019). Resource Management Approaches in Fog Computing: a Comprehensive Review Mostafa. Grid Computing.

Mostafa, N. (2020). Resource Selection Service Based on Neural Network in Fog Environment. Technology and Engineering Systems Journal, 408-417.

Nguyen Cong Luong, Yutao Jiao, Ping Wang, Dusit Niyato, Dong In Kim, and Z. H., \& To. (2020). A MachineLearning-Based Auction for Resource Trading in Fog Computing. Communications Magazine, 58(3), 82-88.

O'Dea, S. (2020). IoT devices in use worldwide 2009-2020. Number of IoT Devices in Use Worldwide from 2009 to 2020. https://www.statista.com/statistics/764026/numberof-iot-devices-in-use-worldwide/

Okay, F. Y. (2019). NESNELERIN INTERNETINDE SIS HESAPLAMA TABANLI VERI KÜMELEME VE YÖNLENDIRME MODELLERI.

POOYAN HABIBI 1, (Student Member, IEEE), M. F., SEPEHR KAZEMIAN2, S. K. 2, \& AND ALBERTO LEONGARCIA 1, (Life Fellow, I. (2020). Fog Computing: A Comprehensive Architectural Survey. IEEE Access, 6910569133.

Qi, Q., \& Tao, F. (2019). A Smart Manufacturing Service System Based on Edge Computing, Fog Computing, and Cloud Computing. IEEE Access, 7, 86769-86777. https://doi.org/10.1109/ACCESS.2019.2923610

S. F. Abedin, M. G. R. Alam, S. M. A. Kazmi, N. H. Tran, D. N. and C. S. H. (2019). Resource Allocation for Ultra-reliable and Enhanced Mobile Broadband IoT Applications in Fog Network. Transactions on Communications, 67(1), 489502.

S. S. Gill, P. Garraghan, R. bUYYA. (2019). ROUTER: Fog enabled cloud based intelligent resource management approach for smart home IoT devices. The Journal of Systems and Software, 154, 125-138 Contents.

Sahni, Y., Cao, J., Zhang, S., \& Yang, L. (2017). Edge Mesh: A New Paradigm to Enable Distributed Intelligence in Internet of Things. In IEEE Access (Vol. 5, pp. 1644116458). https://doi.org/10.1109/ACCESS.2017.2739804

Shah-Mansouri, H., \& Wong, V. W. S. (2018). Hierarchical fogcloud computing for IoT systems: A computation offloading game. In IEEE Internet of Things Journal (Vol. 5, Issue 4, pp. 3246-3257) https://doi.org/10.1109/JIOT.2018.2838022

Shahzadi1, R., \& , Ambreen Niaz1, Mudassar Ali1, *, Muhammad Naeem2 , Joel J.P.C. Rodrigues3, 4, 5, 6 , Farhan Qamar1, S. M. A. (2019). Three tier fog networks: Enabling IoT/5G for latency sensitive applications. In China Communications (Vol. 16, Issue 3, pp. 1-11). https://doi.org/10.12676/j.cc.2019.03.001 
Shi, W., Cao, J., Zhang, Q., Li, Y., \& Xu, L. (2016a). Edge Computing: Vision and Challenges. In IEEE Internet of Things Journal (Vol. 3, Issue 5, pp. 637-646). https://doi.org/10.1109/JIOT.2016.2579198

Shi, W., Cao, J., Zhang, Q., Li, Y., \& Xu, L. (2016b). Edge Computing: Vision and Challenges. IEEE Internet of Things Journal, 3(5), 637-646. https://doi.org/10.1109/JIOT.2016.2579198

Stankovic, J. A. (2014). Research Directions for the Internet of Thing. Internet of Things Journal, 1(1), 3-9.

Sultan, N. (2010). Cloud computing for education: A new dawn? International Journal of Information Management, 30(2), 109-116. https://doi.org/10.1016/j.ijinfomgt.2009.09.004

Tiscornia, O. M., Hamamura, S., De Lehmann, E. S., González, E., Vaccaro, M. I., Otero, G., Cerini, C., \& Waisman, H. (1999). LA Inervación Autonómica Gastro-Entero-BilioPancreática: El concepto de "pista" plexual entérica. In Prensa Medica Argentina (Vol. 86, Issue 2, pp. 129-139).

Tuysuz, M. F. (2019). Deneyim Kalitesi odaklı Akıllı Işsbirlikçi Çoklu-erişimli Uç Hesaplama Çerçevesi QoE-based Smart Cooperative Multi-access Edge Computing Framework Deneyim Kalitesi odaklı Akıllı Işbirlikçi Çoklu-erişimli Uç Hesaplama QoE-based Smart Cooperative Multi-acces. 4(3), 8-18.

Van Os, H. J. A., Mulder, I. A., Van Der Schaaf, I. C., Van Walderveen, M. A. A., Kapelle, L. J., Ferrari, M. D., Algra, A., \& Wermer, M. J. H. (2015). The Internet of Things for Health Care: A Comprehensive Survey. In International Journal of Stroke (Vol. 10, p. 217).

Vaquero, L. M., Rodero-Merino, L., Caceres, J., \& Lindner, M. (2008). A Break in the Clouds: Towards a Cloud Definition. Computer Communication Networks.

W. Wang, Q. W. and K. S. (2016). Multimedia Sensing as a Service (MSaaS): Exploring Resource Saving Potentials of at Cloud-Edge IoTs and Fogs. Internet of Things Journal, 4(7), 487-495.

Wang, L., Jiao, L., Li, J., Gedeon, J., \& Muhlhauser, M. (2019). MOERA: Mobility-Agnostic online resource allocation for edge computing (sayfa 7 de kaldim). IEEE Transactions on Mobile Computing, 18(8), 1843-1856. https://doi.org/10.1109/TMC.2018.2867520

Xu, X., Fu, S., Cai, Q., Tian, W., Liu, W., Dou, W., Sun, X., \& Liu, A. X. (2018). Dynamic Resource Allocation for Load Balancing in Fog Environment. In Wireless Communications and Mobile Computing (Vol. 2018). https://doi.org/10.1155/2018/6421607

Y. Gu, Z. Chang, M. Pan, L. S. and Z. H. (2018). Joint Radio and Computational Resource Allocation in IoT Fog Computing. Transactions on Vehicular Technology, 67(8), 7475-7484.

Y. Liu, M. Peng, G. Shou, Y. C. and S. C. (2020). Toward Edge Intelligence: Multiaccess Edge Computing for $5 \mathrm{G}$ and Internet of Things. Internet of Things Journal, 7(8), 67226747.

Yang, Y., Wang, K., Zhang, G., Chen, X., Luo, X., \& Zhou, M. T. (2018). MEETS: Maximal Energy Efficient Task Scheduling in Homogeneous Fog Networks. In IEEE Internet of Things Journal (Vol. 5, Issue 5, pp. 4076-4087). https://doi.org/10.1109/JIOT.2018.2846644

Yu, W., Liang, F., He, X., Hatcher, W. G., Lu, C., Lin, J., \& Yang, X. (2017). A Survey on the Edge Computing for the Internet of Things. In IEEE Access (Vol. 6, pp. 6900-6919). Institute of Electrical and Electronics Engineers Inc. https://doi.org/10.1109/ACCESS.2017.2778504

Zanella, A., Bui, N., Castellani, A., Vangelista, L., \& Zorzi, M. (2014). Internet of things for smart cities. In IEEE Internet of Things Journal (Vol. 1, Issue 1, pp. 22-32). https://doi.org/10.1109/JIOT.2014.2306328 